\section{Trastuzumab bleibt Therapie der Wahl bei Brustkrebs - auch nach Progression}

\author{
Frauen mit metastasiertem HER2-positivem Brustkrebs, der unter oder kurz \\ nach einer Trastuzumab-Therapie fortschreitet, profitieren nicht von der Um- \\ stellung auf Afatinib. Dieses könnte prognostisch sogar von Nachteil sein.
}

\begin{abstract}
W arum HER2-positive Mammakarzinome unter Trastuzumab resistent gegen den Antikörper werden, ist unklar. Vermutlich führt eine längerfristige Behandlung zur vermehrten Expression von EGFR und HER3, und die Ausbildung von HER2/HER3-Heterodimeren spielt eine wichtige Rolle. Um die Resistenz zu überwinden, könnte es daher sinnvoll sein, Wirkstoffe mit einer breiteren Hemmung von ErbB-Rezeptoren einzusetzen. Diese Hypothese prüfte ein internationales Team um Nadia Harbeck vom Brustzentrum der Universität München in der Studie LUX-Breast 1: 508 Frauen, die nach Trastuzumab progredient waren, wurden 2:1 randomisiert, den HER2-Tyrosinkinasehem-
\end{abstract}

mer (TKI) Afatinib oder Trastuzumab, zu erhalten - jeweils in Kombination mit Vinorelbin.

Die Aufnahme von Patientinnen wurde vorzeitig gestoppt. Denn nach median 9,3 Monaten nur $38 \%$ der Patientinnen unter Afatinib, aber $43 \%$ unter Trastuzumab ohne Progression überlebten. Das mediane progressionsfreie Überleben betrug 5,5 bzw. 5,6 Monate. Ein objektives Ansprechen - vollständig oder partiell - fand sich bei 46 versus $47 \%$. Beim Gesamtüberleben schnitt die Afatinib-Gruppe mit median 19,6 versus 28,6 Monaten gegenüber Trastuzumab deutlich schlechter ab. Diese Ergebnisse können allerdings durch die Anschlussbehandlungen beeinflusst worden sein.
Die Behandlung mit dem TKI war zudem mit mehr Nebenwirkungen belastet. $36 \%$ der Patientinnen (vs. 26\% unter Trastuzumab) hatten schwere Nebenwirkungen, sie führten bei $15 \%$ (vs. $7 \%$ ) zum Therapieabbruch. Die häufigsten unerwünschten Wirkungen vom Grad $\geq 3$ waren Neutropenie (56 vs. $60 \%$ ), Leukopenie (19 vs. $21 \%$ ) und Diarrhö (18 vs. $0 \%$ ).

Fazit: Das Resultat steht in einer Linie mit zwei Studien mit dem TKI Lapatinib: Weder in MA.31 noch in CEREBEL hatte er einen Vorteil erreicht. „Trastuzumab-basierte Therapien bleiben damit Therapie der Wahl für Patientinnen mit metastasiertem HER2-positivem Brustkrebs, wenn Trastuzumab versagt hat", so das Fazit der Forscher. Beate Schumacher

Harbeck $\mathrm{N}$ et al. Afatinib plus vinorelbine versus trastuzumab plus vinorelbine in patients with HER2-overexpressing metastatic breast cancer who had progressed on one previous trastuzumab treatment (LUX-Breast 1): an open-label, randomised, phase 3 trial. Lancet Oncol. 2016; 17(3):357-66.

\title{
Brustkrebs: Wann sind Dosisreduktionen häufig?
}

\section{Ziel dieser Analyse war es zu unter- suchen, inwiefern Symptome der Erkrankung die Anwendung einer Krebstherapie behindern.}

ymptome der Krebserkrankung und $\checkmark$ Nebenwirkungen der Behandlung sind oft die Hauptgründe für Verzögerungen, Dosisreduktionen oder Abbrüche der Therapie. Eine suboptimale Behandlung kann aber lebensbedrohliche Rezidive mit einer Verkürzung des Lebens nach sich ziehen. Der Zusammenhang zwischen Symptomen und Beeinträchtigungen der täglichen Aktivitäten sowie Dosisreduktionen und Therapieabbrüchen könnte wichtige Hinweise für die Durchführung von Verhaltenstherapien liefern.

In der vorliegenden Analyse wurden sekundär die Daten aus einer randomisierten klinischen Studie zu Reflexzonenmassage zum Management von Symptomen unter einer Chemo-, Radio- oder zielgerichteten Therapie bei Frauen mit fortgeschrittenem Mammakarzinom untersucht. 385 Frauen waren in der Studie randomisiert der Reflexzonenmassage, einer „Fußmanipulation“ oder der Standardversorgung zugeteilt worden. Zu Studienbeginn sowie in den Wochen 5 und 11 waren sie in Telefoninterviews zu ihren Symptomen befragt worden. Die Angaben zu Therapieunterbrechungen und Metastasierung stammten aus medizinischen Dokumentationen.

Bis Woche 5 hatten $13 \%$ der Patientinnen die Therapie verzögert oder die Dosis reduziert, zwischen Woche 5 und $115 \%$, die Therapie abgebrochen hatten 8 bzw. 6\%. Die häufigsten Gründe für Dosisreduktionen, Therapieverzögerungen und -abbrüche waren Symptome, Veränderungen der Laborwerte, Infektionen, die Entscheidung der Patientin, Klinikaufenthalte und Krankheitsprogression. Allerdings waren die Richtungen der Assoziationen unterschiedlich:
Bei Patientinnen ohne Fernmetastasen bestand eine negative Assoziation zwischen Symptomschwere, insbesondere der Schmerzen, und der Wahrscheinlichkeit einer Therapieverzögerung oder Dosisreduktion. Anders bei Patientinnen mit Fernmetastasen: Bei ihnen stieg die Wahrscheinlichkeit einer Therapieverzögerung oder Dosisreduktion mit zunehmender Symptomschwere. Vor allem das Ausmaß der Schmerzen war hier ein starker Prädiktor. Möglicherweise haben Ärzte unterschiedliche Haltungen zu Dosisreduktionen in Abhängigkeit von der Metastasierung. Auf die Rate an Therapieabbrüchen hatten weder Symptomschwere noch Metastasierung signifikanten Einfluss.

Fazit: Symptome der Krebserkrankung können die Durchführung der Therapie in unterschiedlichem Maß beeinträchtigen.

Judith Neumaier

Wyatt G et al. Chemotherapy interruptions in relation to symptom severity in advanced breast cancer. Support Care Cancer. 2015;23(11): 3183-91. 